\title{
Application of Electrical Resistance Tomography in an Oscillatory Baffled Column for Gas-Liquid Two-Phase Flow
}

\author{
A. A. Hamzah ${ }^{1,2}$, A. R. Ruzairi ${ }^{1,3,{ }^{*}}$, M.S. Takriff ${ }^{4}$, E.J. Mohamad ${ }^{1}$, \\ J. Pusppanathan ${ }^{5}$, I. N. Azman ${ }^{2}$, M.S. Mohd Ghazali ${ }^{2}$, M.Z. Sabri ${ }^{2}$, \\ K.F. Mohrab², F. Ghafar ${ }^{2}$, M.N. Zahari
}

${ }^{1}$ Faculty of Electrical and Electronic Engineering, Universiti Tun Hussein Onn, 86400 Batu Pahat, Johor, MALAYSIA

${ }^{2}$ Section of Chemical Engineering Technology, Universiti Kuala Lumpur Malaysian Institute of Chemical and

Bioengineering Technology (UniKL MICET), 78000, Alor Gajah, Melaka, MALAYSIA

${ }^{3}$ Faculty of Electrical Engineering, Universiti Teknologi Malaysia, 81310, Skudai, Johor, MALAYSIA

${ }^{4}$ Department of Chemical \& Process Engineering, Faculty of Engineering \& Built Environment, 43600, UKM, Bangi, MALAYSIA

${ }^{5}$ Faculty of Biosciences and Medical Engineering, Universiti Teknologi Malaysia, 81310, Skudai, Johor, MALAYSIA

DOI: https://doi.org/10.30880/ijie.2019.11.06.013

Received 15 March 2018; Accepted 15 April 2019; Available online 12 September 2019

\begin{abstract}
Oscillatory baffled column (OBC) has a high potential for industrial application especially contacting gas and liquid phase. However, there are still lacks of research regarding the dynamics of gas-liquid phase specifically on the visualization of internal flow. Electrical Resistance Tomography (ERT) technique has capabilities to visualize mixing process internally without invading the flow. The objective of this study is to investigate the gas-liquid mixing distribution in an OBC by applying tomography technique model ITS P2000 of ERT system. The parameters investigated are phase distribution, gas hold up and power density. The experimental work was carried out in an OBC with a diameter of $145 \mathrm{~mm}$ and $720 \mathrm{~mm}$ height. The column is consisted of four planes of sixteen stainless steel ERT electrodes. The results of the research have shown that $70 \mathrm{~mm}$ of oscillation amplitude and $2 \mathrm{~Hz}$ of oscillation frequency give the best result with $73 \%$ of gas hold up and $12433.39 \mathrm{~W} / \mathrm{m}^{3}$.
\end{abstract}

Keywords: Conductivity Distribution, Electrical Resistance Tomography, Gas Hold Up, Oscillatory Baffled Column, Power Density

\section{Introduction}

There are numbers of industrial operations which deal with gases and liquids processes. The gases and liquids processes in the industrial operations are such as oxidation, biological fermentations and hydrogenation. The objective of such processes including of gas-liquid phase is to agitate the phase mixture to generate a dispersion of gas bubbles in a continuous liquid phase. The simplest equipment used in the gas-liquid process are stirred tank and bubble column. However, the ordinary equipment has faced many problems and shortcomings in the processing industry. Takriff has listed some of the weaknesses of existing mixing technologies such as higher operating costs, higher shear stress, limited to one phase and need a spacious site [1]. 
Oscillatory Baffled Column (OBC) is a new mixing technology that had been reported numerously for its promising method in mixing process. In the late 1980's and 1990's, numbers of research have been conducted to characterize OBC's and illustrate their strengths on mixing process. Up till now OBC has been used in wide of applications such as ozone-water mass transfer [2], synthesis of isobornyl acetate [3], biodiesel production [4], microalgae and [5] which shows the reactor is capable to replace traditional mixing equipment. OBC's could exhibit highly plug flow conditions at low frequencies and amplitudes and could also provide good global mixing characteristics and proven that the oscillatory flow successfully increase mass transfer and heat transfer [6]. The fluid mechanical condition for OBC system is governed by three dimensionless parameters which are Reynolds number, oscillatory Reynolds number and Strouhal number. Reynolds number is the ratio of inertial forces to viscous forces flow. Oscillatory Reynolds numbers reflects the oscillating movement applied to the flow system while Strouhal number illustrates the effectiveness of the channel diameter ratio to the amplitude of the oscillation is imposed to the flow.

$$
\begin{gathered}
\operatorname{Re}_{n}=\frac{u D \rho}{\mu} \\
\operatorname{Re}_{o}=\frac{2 \pi f x_{o} \rho D}{\mu} \\
S_{t}=\frac{D}{4 \pi x_{o}}
\end{gathered}
$$

$u$ is velocity, $D$ is the Column diameter $(\mathrm{m}), \rho$ is the Fluid density $\left(\mathrm{kgm}^{-3}\right), \mu$ is the Fluid viscosity $\left(\mathrm{kgm}^{-1} \mathrm{~s}^{-1}\right), x_{o}$ is the Oscillatory amplitude $(\mathrm{m})$ and $f$ is the Oscillatory frequency $(\mathrm{Hz})$.

To date, there is no valid technique to accurately characterize two-phase flow phenomena especially gas-liquid system. A new approach sensor technique has been developed to achieve even more accurate measurement with noninvasive and non-disturbance collecting information data. The established techniques are laser, ultrasonic, and tomography. One type of attractive technique in research is tomography. Tomography system has a capability to dismantling the complexity of the internal flow and optimse the process flow without needing to interfere that flow [7]. One of the attractive tomography modalities is eletrical resistance tomography (ERT) which capable to measure and monitor the parameter of multi-phase flow online. Besides that, it is a powerful tool for direct analysis and fast in collecting data acquisition. According to Jin et al. [8], ERT can be considered as a robust tomographic technique due to its speed, cost and suitability for different vessels size. Therefore, some researchers also have put an effort to apply this technique in their research such as trombus vizualization [9] deffect visulazation [10], bioprocessing industries [11] and mixing process [12-14]. However, the application of ERT in the investigation of multiphase flow in an OBC is still at early stage. Issa et al, [15] was the first researcher who used ERT to measure velocity in an OBC. Usually computational fluid mechanics (CFD) is used to characterized mixing process in OBC system [16-17].

This work aims to investigate the mixing process for gas-liquid phase in an oscillatory baffled column by using electrical resistance tomography. Four planes with 16 electrodes per plane were fitted on the OBC to identify the conductivity distributions, calculate the gas hold up by using Maxwell relationship and the effectiveness of mixing process was determined by applying the power density correlation.

\section{Methodology}

The system used contained an oscillatory baffled column within 145-mm-ID and fitted with 16 stainless steel electrodes at each plane at the column wall. Four planes were used for this column. Gas was channeled via 2 mm nozzle at the bottom of the column. P2000 ERT is made up of three components which are the sensing electrode arrays, data acquisition system (DAS) with process unit and image reconstruction with parameter extract unit. Operating system was initiated by inducing electrical current between one pair of electrodes and differential potentials for all remaining pairs are measured (can be seen clearly in Fig. 1) [18]. The functions of DAS are to collect the real-time instantaneous signals output from sensing electrode arrays and to reflect the measured field to 2D or 3D distribution [19]. With 20 frames per second data acquisition speed and $9.6 \mathrm{kHz}$ frequency, P2000 ERT system can collect 104 voltage values per data frame. Two and three dimensional images which reflect the dynamic variation of measured field will be constructed by the image reconstruction algorithm built in P2000 ERT system. Fig. 1 shows the design of 4 planes-16 electrodes of ERT sensor fitted on OBC column. Sensor was placed $70 \mathrm{~mm}$ distance from baffled at both top and bottom to visualize the vortex created by up-flow (P2 and P4) and down-flow (P1 and P3) respectively. 
(a)

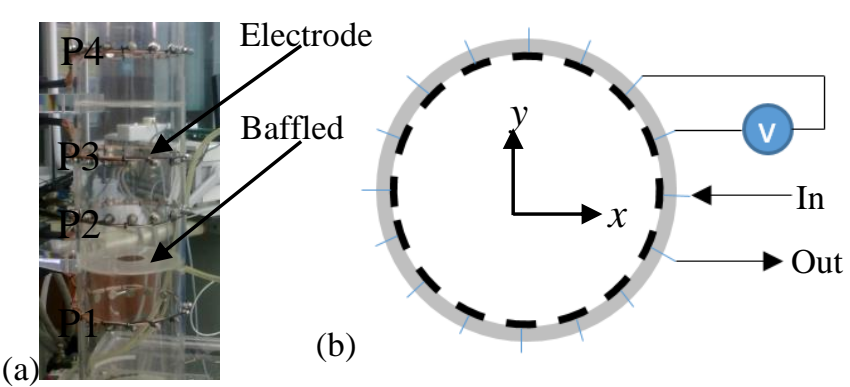

Fig. 1 - Sensor design (a) 4 plane-16 electrodes (b) cross-sectional view

The experiment run was intended to analyze the gas dispersion with piston movement and without gas and piston movement. Without gas test is used to be a reference data. The gas flow rate for this experiment was $6 \mathrm{~L} / \mathrm{min}$. Oscillation amplitude, $x_{o}=50,60$ and $70 \mathrm{~mm}$ and coupled with 1 and $2 \mathrm{~Hz}$ oscillation frequency. Tap water was used as the continuous phase while air was introduced at the bottom of the column. Table 1 shows the electrical properties of materials used. The water temperature was maintained under ambient conditions, at about $27^{\circ} \mathrm{C}$. Fig. 2 shows the column fitted with sensing array and ERT P2000 system in schematic rig.

Table 1 - Electrical properties of material used.

\begin{tabular}{lcc}
\hline Properties & Gas & Tap water \\
\hline Conductivity $(\mathrm{S} / \mathrm{m})$ & $10-15-10^{-9}$ & $5 \times 10^{-4}-5 \times 10^{-2}$ \\
Resistivity $(\Omega . \mathrm{m})$ & $10^{9}-10^{15}$ & $2 \times 10^{1}-2 \times 10^{3}$ \\
\hline
\end{tabular}

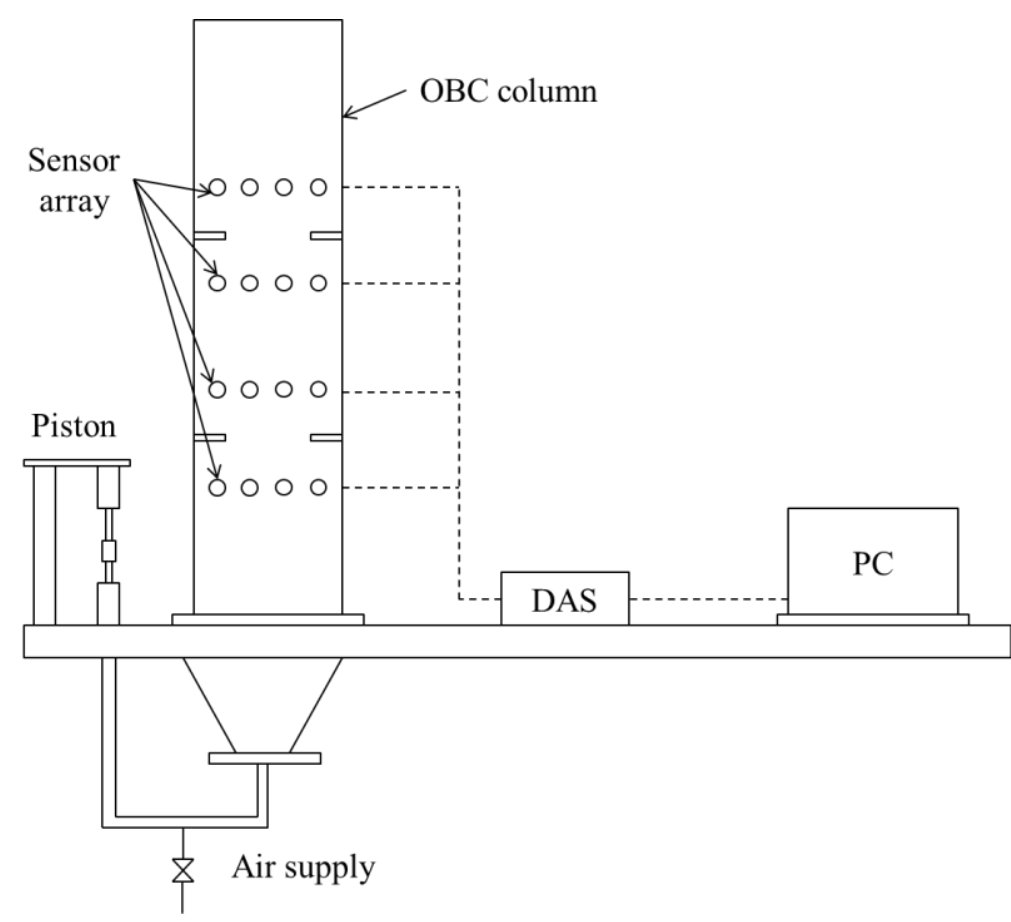

Fig. 2 - Sensing array and schematic rig ERT):

For ERT, gas hold-up is calculated by applying the Maxwell relationship (the conductivity data obtained from

$$
\varepsilon_{g, i}=\frac{2 \sigma_{1}+\sigma_{2}-2 \sigma_{m c}-\sigma_{m c} \sigma_{2} / \sigma_{1}}{\sigma_{m c}-\sigma_{2} / \sigma_{1}+2\left(\sigma_{1}-\sigma_{2}\right)}
$$

where $\sigma_{1}$ is conductivity of the first phase, $\sigma_{2}$ is conductivity of the second phase and $\sigma_{m c}$ is mixture conductivity. If the second phase is consisted of non-conductive material, the above equation can be simplified into: 


$$
\varepsilon_{g, i}=\frac{2 \sigma_{1}-2 \sigma_{m c}}{2 \sigma_{1}+\sigma_{m c}}
$$

The input per unit volume of the power density, $\mathrm{P} / \mathrm{V}$, can be related to the dispersion into the gas liquid mixing according to equation 6 for oscillatory baffled column [15]. Higher power density values imply that the mixing process is better.

$$
\left(\frac{P}{V}\right)_{O B C}=\sqrt[c]{\frac{\varepsilon_{g}}{a_{2}}}
$$

Where $a_{2}=0.0002$ and $c=0.87$ for OBC [16].

\section{Results and discussions}

\subsection{Conductivity Distribution}

Image reconstruction algorithm used in this system to reconstruct conductivity distributions is Linear Back Projection (LBP) which is non-iterative algorithms that offers fast processing times comparing to iterative algorithms and has capabilities to show the phase different of gas and liquid for the experimental interest. Fig. 3 as below shows the contours of conductivity distribution images for oscillation amplitude, $x_{o} 50,60$ and $70 \mathrm{~mm}$ coupled with 1 and $2 \mathrm{~Hz}$ oscillation frequency, $f$ for four planes. Conductivity image is labelled in red for the area which was attended by more conductive phase. For the dispersed phase such as the gas which is non-conductive, it was marked with blue color. Four planes (P1 to P4) with 16 electrodes per plane were turned on to detect any changes in an OBC. Experiments were carried out by taking 300 images to ensure uniform gas-liquid mixing prior to observed data. These tomograms clearly show that, at $50 \mathrm{~mm}$ oscillation amplitude coupled with 1 and $2 \mathrm{~Hz}$ oscillation frequency, the gases were not dispersed thoroughly in OBC. The water phase marked with red color concentrated at the wall shows that mixing was not going well with this configuration of amplitude-frequency. When $x_{o}$ was increased to $60 \mathrm{~mm}$, the yellowish color was present at the plane 2, 3 and 4. On the other hand, at plane 1, gas and liquid were still separated as mixing process was not well. At the $x_{o}=70 \mathrm{~mm}$, the yellowish and greenish color was present in the tomograms showing that the gas liquid mixing was going well in the column.

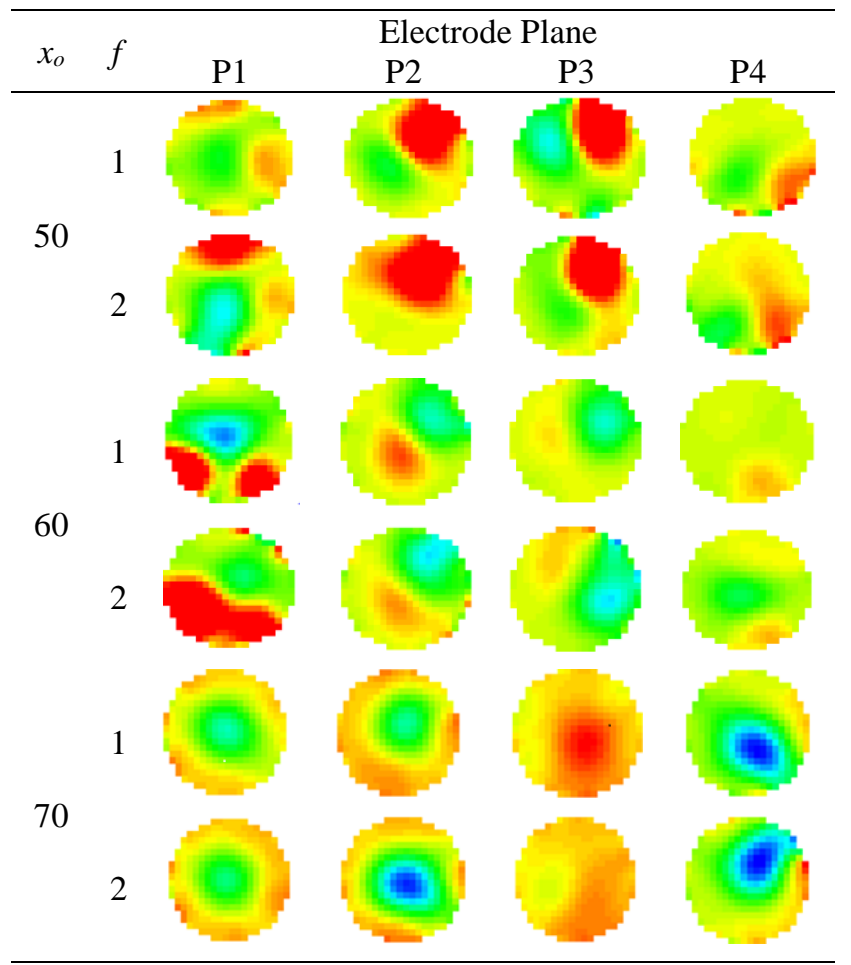

Fig. 3 - Conductivity distributions 
Fig. 3 also shown the optimal mixing process occurred for $x_{o}=70 \mathrm{~mm}$ at plane no 2 and 3 with yellowish to reddish color were dominant. At both planes, 2 baffles were fitted as a special design of OBC in purpose to enhance the mixing intensity. Gas and fluid were pushed upward by piston and increase the acceleration of fluid through the baffle openings and formed a vortex in the rear baffle. When the deceleration of the fluid begins (piston moving down), this vortex will be pushed to the center of the baffles and good mixing will occur in the space between the baffle plates. Formation and coalescence of vortex encounters are the main mechanism that induce the movement and increase the radial velocity of fluid in the space between the baffle plates further produce a perfect mixing. The cycle of vortex formation and high velocity in the radial direction will provide uniform mixing in the area between the baffle and cumulatively along the column.

\subsection{Gas Hold-up}

Gas hold up is often used to reflect the volume occupied by the gas phase. The value of gas hold up for a container that is used for mixing gas-liquid phase can be used as a qualitative indicator of the rate of mass transfer. Gas holdup is also an important factor that gives rise to pressure variation and thus liquid recirculation. and determined by the ratio of gas phase to overall volume [20]. The higher overall value of gas holdup will state that the mixing process for gas-liquid is preferable where more gas bubbles dispersed into the liquid phase [21-22].

By using equation (5), conductivity data from ERT can be used to calculate the value of gas hold up in an OBC. Table 2 shows the value of gas hold up at different oscillation amplitude coupled with oscillation frequency.

Table 2 - Gas hold up for different oscillation amplitude and frequency

\begin{tabular}{ccc}
\hline Amplitude $\boldsymbol{x}_{\boldsymbol{o}} \mathbf{( m m )}$ & Frequency $\boldsymbol{f}(\mathbf{H z})$ & Gas Hold up $(\boldsymbol{\%})$ \\
\hline \multirow{2}{*}{50} & 1 & 72.5 \\
& 2 & 72.6 \\
\multirow{2}{*}{60} & 1 & 72.7 \\
& 2 & 72.8 \\
\multirow{2}{*}{70} & 1 & 72.9 \\
& 2 & 73.0 \\
\hline
\end{tabular}

The higher value for gas hold up is $73 \%$ at $x_{o}=70 \mathrm{~mm}$ and $f=2 \mathrm{~Hz}$. From the results obtained, it showed that gas hold up increased with an increase in the amplitude of specific oscillation frequency. The results are in agreement with [23] findings. This is because the function of the oscillation amplitude and frequency increased the rate of formation and coalescence of the resulting vortex. Formation and coalescence of vortex is the primary mechanism for the mixing in an $\mathrm{OBC}$ that it is directly proportional to the rate of mixing.

\subsection{Power Density}

Table 3 summarizes the power density for average gas hold up obtained. From the calculation, $\left(x_{o}=70 \mathrm{~mm} f=2 \mathrm{~Hz}\right)$ gives a higher values which is $12433.39 \mathrm{~W} / \mathrm{m}^{3}$ compared to others. Higher power number lead to a better mixing intensity, result a higher gas hold up obtained.

Fig. 4 and 5 shows effect of amplitude on the gas hold up and power density for oscillation frequency $1 \mathrm{and} 2 \mathrm{~Hz}$ respectively. The gas hold up and power density linearly increased with increasing of amplitude value. Amplitude and frequency is the main parameter which control the efficiency of mixing process in an OBC system. The best combination of amplitude and frequency will produce increase and optimize mixing intensity. Normally higher value of amplitude and frequency selected produced the best mixing condition. However, too higher combination selected will lead to channeling flow which the gas bubble only passing the annular part of baffle and create dead zone surrounded baffle on the OBC system.

Table 3 - Power Density

\begin{tabular}{ccc}
\hline Amplitude $\boldsymbol{x}_{\boldsymbol{o}}(\mathbf{m m})$ & Frequency $\boldsymbol{f}(\mathbf{H z})$ & Power Density, $(\mathbf{P} / \mathbf{V})\left(\mathbf{W} / \mathbf{m}^{\mathbf{3}}\right)$ \\
\hline \multirow{2}{*}{50} & 1 & 12335.55 \\
& 2 & 12355.11 \\
60 & 1 & 12374.68 \\
& 2 & 12394.24 \\
\multirow{2}{*}{70} & 1 & 12413.81 \\
& 2 & 12433.39 \\
\hline
\end{tabular}

The results presented in the Figure 4 and 5 shows a little different value of gas hold up and power density changes with the combination of amplitude from 50 to $70 \mathrm{~mm}$ with 1 and $2 \mathrm{~Hz}$ frequency. The higher value of gas hold up and power density is on the $70 \mathrm{~mm}$ amplitude couple with $2 \mathrm{~Hz}$ frequency. However so far, the value is not too differing 
with the combination of $70 \mathrm{~mm}$ of amplitude and $1 \mathrm{~Hz}$ of frequency. Based on the tomogram obtained, the best condition for a diameter of $145 \mathrm{~mm}$ and $720 \mathrm{~mm}$ height $\mathrm{OBC}$ is $70 \mathrm{~mm}$ of oscillation amplitude coupled with $1 \mathrm{~Hz}$ of frequency. The best mixing was occurred when the gasses dispersed throughout the column. This condition was shown by yellowish color in tomogram for all planes except plane 4 which placed near to the end of the column. The application of ERT for characterizes gas-liquid mixing in an OBC system successfully visualizes internal flow by providing cross section image and possibly to replace the traditional method which depends on the simulation work. The combination between internal visualization by ERT and calculation of gas hold up and power density will increase the efficiency of the characterization of mixing process in an OBC system.

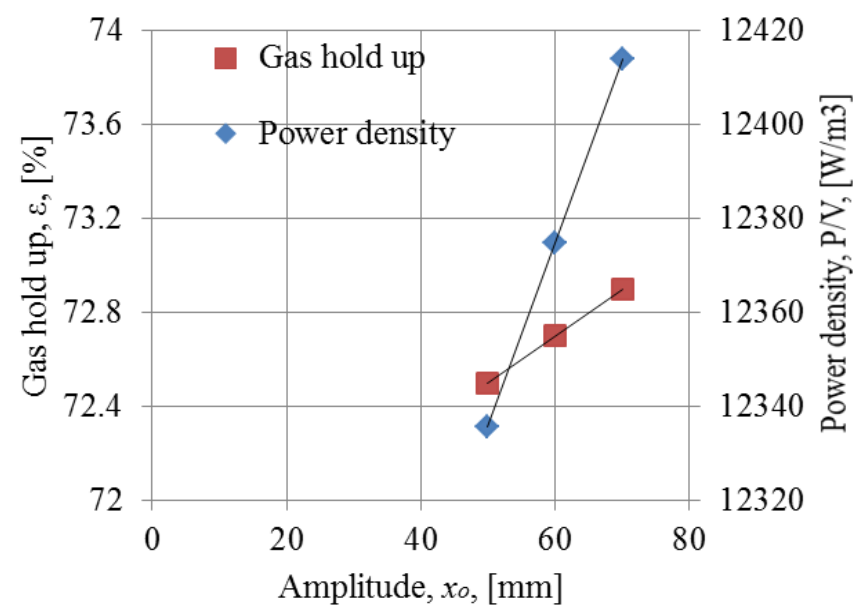

Fig. 4 - The gas hold up and power density for frequency $1 \mathrm{~Hz}$

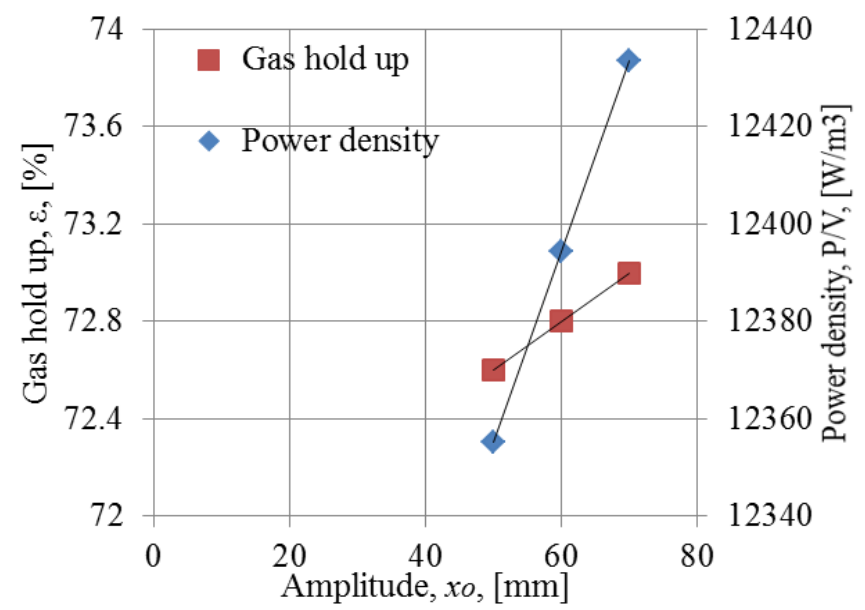

Fig. 5 - The gas hold up and power density for frequency $2 \mathrm{~Hz}$

\section{Conclusions}

The results presented in this paper have shown that ERT was capable to analyze the mixing process for gas-liquid phase in an OBC. The method presented as a better alternative to quantify the mixing process in an OBC system rather than simulation method. The tomographic images of conductivity distribution have qualitatively characterized gas-liquid dispersion in an OBC with the respective oscillation amplitude and frequency. Oscillation amplitude $70 \mathrm{~mm}$ coupled with $1 \mathrm{~Hz}$ of oscillation frequency give the best results compared to the others based on gas hold up and power density analysis. The application of ERT on the OBC is still in the early stage. More parameters should be investigated such as residence time, gas flow rate and design of baffled itself. At the same time, iterative algorithm can be introduced to get the better ERT image reconstruction. The new generation of an OBC can replace the traditional equipment such as agitated vessel in mixing process because of their abilities. 


\section{References}

[1] M. S. Takriff. (2006). Pencampuran aliran berayun. Bangi: Universiti Kebangsaan Malaysia.

[2] A. Al-abduly, P. Christensen, A. Harvey, and K. Zahng. (2014). Characterization and optimization of an oscillatory baffled reactor ( OBR ) for ozone-water mass transfer. Chem. Eng. Process. Process Intensif. 84: 8289.

[3] H. Zheng, Z. Yan, S. Chu, and J. Chen. (2018). Continuous synthesis of isobornyl acetate catalyzed by a strong acid cation exchange resin in an oscillatory fl ow reactor. Chem. Eng. Process. Process Intensif. 134:1-8.

[4] J. F. García-martín, C. C. Barrios, F. Al, and A. Dominguez-s. (2018). Biodiesel production from waste cooking oil in an oscillatory fl ow reactor. Performance as a fuel on a TDI diesel engine.125:546-556.

[5] M. S. R. Abbott, C. M. Brain, A. P. Harvey, M. I. Morrison, and G. Valente. (2015). Liquid culture of microalgae in a photobioreactor ( PBR ) based on oscillatory baf fl ed reactor ( OBR ) technology - A feasibility study. Chem. Eng. Sci.. 138: 315-323.

[6] S. M. R. Ahmed, A. N. Phan, and A. P. Harvey. (2018). Mass transfer enhancement as a function of oscillatory ba ffl ed reactor design. Chem. Eng. Process. Process Intensif.130: 229-239.

[7] E. J. Mohamad, R. A. Rahim, M. H. F. Rahiman, H. L. M. Ameran, Y. A. Wahab, and O. M. F. Marwah. (2016). Analysis of crude palm oil composition in a chemical process conveyor using Electrical Capacitance Tomography. Flow Meas. Instrum. 50:57-64.

[8] H. Jin, M. Wang, and R. A. Williams. (2007). Analysis of the rise velocity of bubble swarm and sauter mean bubble size in bubble columns using electrical resistance tomography Analysis of bubble behaviors in bubble columns using electrical resistance tomography. 130:179-185.

[9] A. Sapkota, T. Fuse, M. Seki, O. Maruyama, M. Sugawara, and M. Takei.(2015). Application of electrical resistance tomography for thrombus visualization in blood. Flow Meas. Instrum.46: 334-340.

[10] H. Ren, K. Tian, S. Hong, B. Dong, F. Xing, and L. Qin. (2019). Visualized investigation of defect in cementitious materials with electrical resistance tomography. Constr. Build. Mater..196: 428-436.

[11] M. A. D. De Rienzo, R. Hou, and P. J. Martin. (2018). Use of electrical resistance tomography ( ERT ) for the detection of biofilm disruption mediated by biosurfactants," Food Bioprod. Process. 110:1-5.

[12] C. Carletti, G. Montante, C. De Blasio, and A. Paglianti. (2016). Liquid mixing dynamics in slurry stirred tanks based on electrical resistance tomography. Chem. Eng. Sci..152:478-487.

[13] D. Malik and L. Pakzad. (2017). Experimental investigation on an aerated mixing vessel through electrical resistance tomography ( ERT ) and response surface methodology ( RSM ) Standard deviation. Chem. Eng. Res. Des. 129: 327-343.

[14] B. K. Singh, A. Quiyoom, and V. V Buwa. (2017). Dynamics of gas-liquid flow in a cylindrical bubble column_ Comparison of electrical resistance tomography and voidage probe measurements. Chem. Eng. Sci.158:124-139.

[15] M. Issa, H. Li, H. I. Schlaberg, M. Wang, and R. A. Williams. (2009). Velocity measurements of multi-phase unsteady flow in oscillatory baffled reactor using electrical resistance tomography. 3rd International Workshop on Process Tomography (IWPT-3).

[16] K. Sutherland, L. Pakzad, and P. Fatehi. (2019). CFD population balance modeling and dimensionless group analysis of a multiphase oscillatory baffled column ( OBC ) using moving overset meshes. Chem. Eng. Sci. 199:552-570.

[17] G. Jimeno, Y. Chu, and X. Ni. (2018). On the evaluation of power density models for oscillatory baffled reactors using CFD. Chem. Eng. Process. Process Intensif.134:153-162.

[18] B. Vadlakonda and N. Mangadoddy. (2018). Hydrodynamic study of three-phase fl ow in column fl otation using electrical resistance tomography coupled with pressure transducers. Sep. Purif. Technol. 203:274-288.

[19] S. Ridzuan, R. Abdul, M. Ha, F. Rahiman, F. Rahman, M. Yunus, and C. Loon. (2014). Electrical resistance tomography: A review of the application of conducting vessel walls. 254:256-264.

[20] M. V Sardeshpande, S. Gupta, and V. V Ranade. (2017). Electrical resistance tomography for gas holdup in a gasliquid stirred tank reactor. Chem. Eng. Sci. 170: 476-490.

[21] M. Wang, A. Dorward, D. Vlaev, and R. Mann. (2000). Measurements of gas - liquid mixing in a stirred vessel using electrical resistance tomography ( ERT ). Chem. Eng. J. 77:93-98.

[22] M. S. Takriff, J. Abdullah, A. A. Hamzah, and A. Ahmad. (2006). ERT Visualization of Gas-Liquid Mixing in an Agitated Vessel. 3rd International Workshop on Process Tomography (IWPT-3), Tokyo, Japan ERT. 1-8.

[23] H. Ahmad Azahari, N. Hasan, T. Mohd Sobri, K. Siti Kartom, J. Abdullah, I. M. Tan, and S. Wah Keng. (2012). Effect of oscillation amplitude on velocity distributions in an oscillatory baffled column ( OBC ). Chem. Eng. Res. Des. 90(8):1038-1044. 